Article

\title{
Fuzzy Logic as a Tool for Assessing Students' Knowledge and Skills
}

\section{Michael Gr. Voskoglou}

School of Technological Applications, Graduate Technological Educational Institute, Patras 223 34, Greece; E-Mail: voskoglou@ teipat.gr or mvosk@ hol.gr; Tel./Fax: +30-2610-328-631

Received: 6 February 2013; in revised form: 21 May 2013/ Accepted: 22 May 2013 /

Published: 31 May 2013

\begin{abstract}
Fuzzy logic, which is based on fuzzy sets theory introduced by Zadeh in 1965, provides a rich and meaningful addition to standard logic. The applications which may be generated from or adapted to fuzzy logic are wide-ranging and provide the opportunity for modeling under conditions which are imprecisely defined. In this article we develop a fuzzy model for assessing student groups' knowledge and skills. In this model the students' characteristics under assessment (knowledge of the subject matter, problem solving skills and analogical reasoning abilities) are represented as fuzzy subsets of a set of linguistic labels characterizing their performance, and the possibilities of all student profiles are calculated. In this way, a detailed quantitative/qualitative study of the students' group performance is obtained. The centroid method and the group's total possibilistic uncertainty are used as defuzzification methods in converting our fuzzy outputs to a crisp number. According to the centroid method, the coordinates of the center of gravity of the graph of the membership function involved provide a measure of the students' performance. Techniques of assessing the individual students' abilities are also studied and examples are presented to illustrate the use of our results in practice.
\end{abstract}

Keywords: fuzzy sets; fuzzy logic; defuzzification; students' assessment

\section{Introduction}

There used to be a tradition in science and engineering of turning to probability theory when one is faced with a problem in which uncertainty plays a significant role. This transition was justified when 
there were no alternative tools for dealing with the uncertainty. Today this is no longer the case. Fuzzy logic, which is based on fuzzy sets theory introduced by Zadeh [1] in 1965, provides a rich and meaningful addition to standard logic. This theory proposed in terms of the membership function operating over the range $[0,1]$ of real numbers. New operations for the calculus of logic were also proposed and showed to be in principle at least a generalization of classic logic [1,2]. Despite the fact that both operate over the same numeric range [0,1], fuzzy set theory is distinct from probability theory. For example, the probabilistic approach yields the natural language statement "there is an $85 \%$ chance that Mary is tall", while the fuzzy terminology corresponds to the expression "Mary's degree of membership within the set of tall people is 0.85 ". The semantic difference is significant: The first view supposes that Mary is or is not tall (still caught in the law of the Excluded Middle); it is just that we only have a $85 \%$ chance of knowing in which set she is in. By contrast, fuzzy terminology supposes that Mary is "more or less" tall, or some other term corresponding to the value of 0.85 . Another immediately apparent difference is that the summation of probabilities of the single subsets (events) of a universal set must equal 1, while there is no such requirement for membership degrees. Further distinctions and differences between probability and fuzziness also exist, arising from the way that the corresponding operations are defined.

The applications which may be generated from or adapted to fuzzy logic are wide-ranging and provide the opportunity for modeling under conditions which are inherently imprecisely defined, despite the concerns of classical logicians. A real test of the effectiveness of an approach to uncertainty is the capability to solve problems which involve different facets of uncertainty. Fuzzy logic has a much higher problem solving capability than standard probability theory. Most importantly, it opens the door to construction of mathematical solutions of computational problems which are stated in a natural language. In contrast, standard probability theory does not have this capability, a fact which is one of its principal limitations. All the above gave us the impulsion to introduce principles of fuzzy logic to describe in a more effective way a system's operation in situations characterized by a degree of vagueness and/or uncertainty (e.g., see [3,4]). In Education, such situations often appear in the cases of learning a subject matter, of problem-solving, of modeling, of analogical reasoning, etc. In fact, students' cognition utilizes in general concepts that are inherently graded and therefore fuzzy. On the other hand, from the teacher's point of view there usually exists vagueness about the degree of success of students in each of the stages of the corresponding didactic situation. As a consequence, our fuzzy model mentioned above finds a lot of applications in the area of education (see $[3,5,6]$, etc.).

The present paper proposes the use of fuzzy logic in assessing students' knowledge and skills. The text is organized as follows: In section 2 we use our general fuzzy framework mentioned above as a tool for students groups' assessment. In section 3 we apply the methods of the "center of gravity" and of the system's total possibilistic uncertainty as defuzzification methods in converting our fuzzy outputs to a crisp number and we present an example illustrating our results in practice. In section 4 we study techniques of students' individual assessment and finally in section 5 we state our conclusions and we discuss our plans for future research. For general facts on fuzzy sets we refer freely to the book of Klir and Folger [7]. 


\section{A Fuzzy Model for Assessing Student Groups' Performance}

One of the problems faced by teachers is the assessment of their students' knowledge and aptitudes. In fact, our society demands not only to educate, but also to classify the students according to their qualifications as being suitable or unsuitable for carrying out certain tasks or holding certain posts. According to the standard methods of assessment, a mark, expressed either with a numerical value within a given scale (e.g., from 0 to 10) or with a letter (e.g., from A to F) corresponding to the percentage of a student's success, is assigned in order to characterize his/her performance. However, this crisp characterization, based on principles of the bivalent logic (yes-no), although it is the one usually applied in practice, it is not probably the most suitable to determine a student's performance. In fact, the teacher can be never absolutely sure about a particular numerical grade characterizing the student's abilities and skills. In contrast, fuzzy logic, due to its nature of including multiple values, offers a wider and richer field of resources for this purpose. Therefore, the application of fuzzy logic that we shall attempt in this section seems to be a valuable tool for developing a framework for the students' assessment.

Let us consider a class of $n$ students, $n \geq 1$ and let us assume that the teacher wants to assess the following students' characteristics: $S_{1}=$ knowledge of a subject matter, $S_{2}=$ problem solving related to this subject matter and $S_{3}=$ ability to adapt properly the already existing knowledge for use in analogous similar cases (analogical reasoning; of course the teacher could choose characteristics different for those mentioned here and may be more than three in total). However, the more are the characteristics chosen for assessment, the more complicated (technically) becomes our model.). Denote by $a, b, c, d$, and $e$ the linguistic labels (fuzzy expressions) of very low, low, intermediate, high and very high success respectively of a student in each of the $S_{i} s$ and set $U=\{a, b, c, d, e\}$.

We are going to attach to each students' characteristic $S_{i}, \mathrm{i}=1,2,3$, a fuzzy subset, $A_{i}$ of $U$. For this, if $n_{i a}, n_{i b}, n_{i c}, n_{i d}$ and $n_{i e}$ denote the number of students that faced very low, low, intermediate, high and very high success with respect to $S_{i}$ respectively, we define the membership function $m_{A i}$ for each $x$ in $U$, as follows:

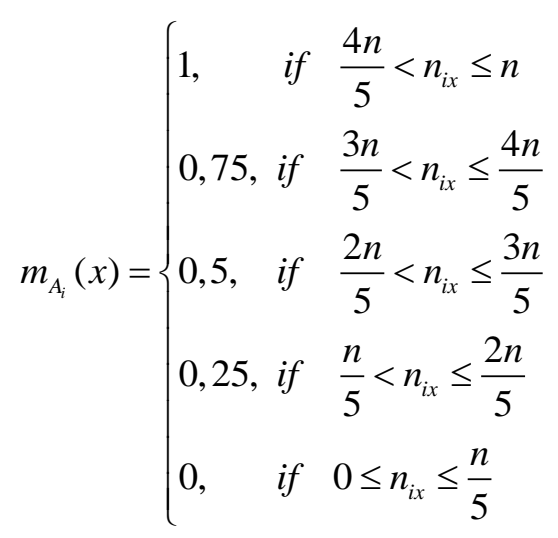

In fact, if one wanted to apply probabilistic standards in measuring the degree of the students' success at each stage of the process, then he/she should use the relative frequencies $\frac{n_{i x}}{n}$. Nevertheless, such an action would be highly questionable, since the $n_{i x} s$ are obtained with respect to the linguist labels of $U$, which are fuzzy expressions by themselves. Therefore, the application of a fuzzy approach by using membership degrees instead of probabilities seems to be the most suitable for this case. 
However, as it is well known, the membership function is usually defined empirically in terms of logical or/and statistical data. In our case the above definition of $m_{A_{i}}$ seems to be compatible with common sense. Then, the fuzzy subset $A_{i}$ of $U$ corresponding to $S_{i}$ has the form:

$$
A_{i}=\left\{\left(x, m_{A i}(x)\right): x \in U\right\}, i=1,2,3 .
$$

In order to represent all possible students' profiles (overall states) with respect to the assessing process we consider a fuzzy relation, say $R$, in $U^{3}$ (i.e., a fuzzy subset of $U^{3}$ ) of the form:

$$
R=\left\{\left(s, m_{R}(s)\right): s=(x, y, z) \in U^{3}\right\} .
$$

For determining properly the membership function $m_{R}$ we give the following definition:

A profile $s=(x, y, z)$, with $x, y, z$ in $U$, is said to be well ordered if $x$ corresponds to a degree of success equal or greater than $y$ and $y$ corresponds to a degree of success equal or greater than $z$.

For example, $(c, c, a)$ is a well ordered profile, while $(b, a, c)$ is not.

We define now the membership degree of a profile $\mathrm{s}$ to be $\mathrm{m}_{\mathrm{R}}(\mathrm{s})=\mathrm{m}_{A_{1}}(\mathrm{x}) \mathrm{m}_{A_{2}}(\mathrm{y}) \mathrm{m}_{A_{3}}(\mathrm{z})$, if $\mathrm{s}$ is well ordered, and 0 otherwise. In fact, if for example the profile $(b, a, c)$ possessed a nonzero membership degree, how it could be possible for a student, who has failed at the problem solving stage, to perform satisfactorily at the stage of analogical reasoning, where he/she has to adapt the existing knowledge for solving problems related to analogous similar cases?

Next, for reasons of brevity, we shall write $m_{s}$ instead of $m_{R}(s)$. Then the probability $p_{s}$ of the profile $\mathrm{s}$ is defined in a way analogous to crisp data, i.e., by $p_{s}=\frac{m_{s}}{\sum_{s \in U^{3}} m_{s}}$. We define also the possibility $r_{s}$ of $s$ to be $r_{s}=\frac{m_{s}}{\max \left\{m_{s}\right\}}$, where $\max \left\{m_{s}\right\}$ denotes the maximal value of $m_{s}$ for all $s$ in $U^{3}$. In other words the possibility of $s$ expresses the "relative membership degree" of $s$ with respect to $\max \left\{m_{s}\right\}$. From the above two definitions it becomes evident that $p_{s}<r_{s}$ for all $s$ in $U^{3}$, which is compatible to the common logic. In fact, whatever is probable it is also possible, but whatever is possible need not be very probable.

Assume now that one wants to study the combined results of the performance of $k$ different groups of students, $k \geq 2$. For this, we introduce the fuzzy variables $A_{1}(t), A_{2}(t)$ and $A_{3}(t)$ with $t=1,2, \ldots, k$. The values of these variables represent fuzzy subsets of $U$ corresponding to the students' characteristics under assessment for each of the $k$ groups; e.g., $A_{l}(2)$ represents the fuzzy subset of $U$ corresponding to the knowledge of a subject matter (characteristic $S_{l}$ ) for the second group $(t=2)$. Obviously, in order to measure the degree of evidence of the combined results of the $k$ groups, it is necessary to define the probability $p(s)$ and the possibility $r(s)$ of each profile $s$ with respect to the membership degrees of $s$ for all groups. For this reason we introduce the pseudo-frequencies $f(s)=m_{s}(1)+m_{s}(2)+\ldots .+m_{s}(k)$ and we define the probability and possibility of a profile $s$ by $p(s)=\frac{f(s)}{\sum_{s \in U^{3}} f(s)}$ and $r(s)=\frac{f(s)}{\max \{f(s)\}}$ respectively, where $\max \{f(s)\}$ denotes the maximal pseudo-frequency. The same method could be applied when one wants to study the combined results of $k$ different assessments of the same student group. 
The above model gives, through the calculation of probabilities and possibilities of all students' profiles, a quantitative/qualitative view of their realistic performance.

\section{Defuzzification Methods}

Defuzzification is the process of producing a quantifiable result in fuzzy logic given fuzzy sets and corresponding membership degrees. Here we shall apply two different approaches for this purpose and we shall compare the corresponding results.

\subsection{The Centroid Method}

A common and useful defuzzification technique is the method of the center of gravity, usually referred as the centroid method. According to this method, given a fuzzy subset $A=\{(x, m(x)): x \in U\}$ of the universal set $U$ of the discourse with membership function

$m: U \rightarrow[0,1]$, we correspond to each $x \in U$ an interval of values from a prefixed numerical distribution, which actually means that we replace $U$ with a set of real intervals. Then, we construct the graph $F$ of the membership function $y=m(x)$. There is a commonly used in fuzzy logic approach to measure performance with the pair of numbers $\left(x_{c}, y_{c}\right)$ as the coordinates of the center of gravity, say $F_{c}$, of the graph $F$, which we can calculate using the following well-known (e.g., see [8]) formulas:

$$
x_{c}=\frac{\iint_{F} x d x d y}{\iint_{F} d x d y}, y_{c}=\frac{\iint_{F} y d x d y}{\iint_{F} d x d y}
$$

Subbotin et al. adapted the centroid method for use with our fuzzy model for the process of learning [9] and they have applied it on comparing students' mathematical learning abilities [10] and for measuring the scaffolding (assistance) effectiveness provided by the teacher to students [11]. More recently, together with Prof. Subbotin we have applied this method in measuring the effectiveness of Case-Based Reasoning Systems [12] and of students' Analogical Reasoning skills [13].

Here we shall apply the centroid method as a defuzzification technique for the student groups' assessment model developed in the previous section. For this, we characterize a student's performance as very low (a) if $\mathrm{y} \in[0,1)$, as low (b) if $\mathrm{y} \in[1,2)$, as intermediate (c) if $\mathrm{y} \in[2,3$ ), as high (d) if $\mathrm{y} \in[3,4)$ and as very high (e) if $\mathrm{y} \in[4,5)$ respectively (these characterizations are usually awarded on the basis of the reports prepared by the students during the course and the results of the progress exams (if any) and the final exam). In this case the graph $F$ of the corresponding fuzzy subset of $U$ is the bar graph of Figure 1 consisting of 5 rectangles, say $F_{i}, i=1,2,3,4,5$, having the lengths of their sides on the $\mathrm{x}$ axis equal to 1 . 
Figure 1. Bar graphical data representation.

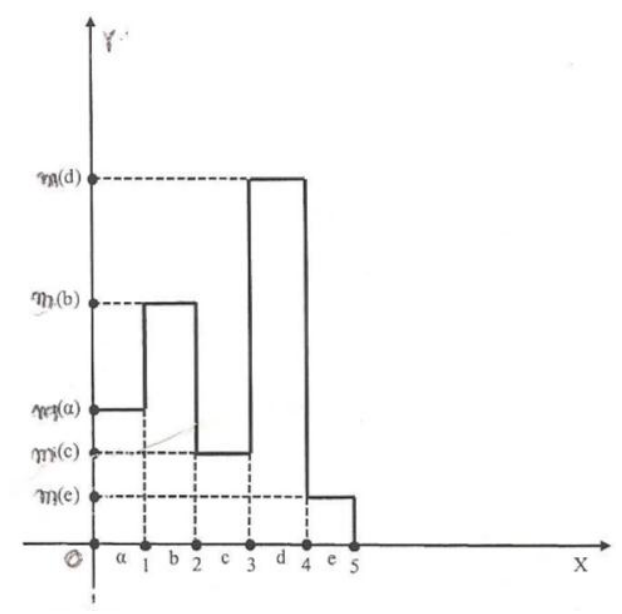

Here $\iint_{F} d x d y$, which is the total area of $\mathrm{F}$, is equal to $\sum_{i=1}^{5} y_{i}$. We also have that $\iint_{F} x d x d y=$ $\sum_{i=1}^{5} \iint_{F_{i}} x d x d y=\sum_{i=1}^{5} \int_{i=1}^{i}\left[\int_{0}^{y_{i}} x d y\right] d x=\sum_{i=1}^{5} y_{i} \int_{i=1}^{i} x d x=\frac{1}{2} \sum_{i=1}^{5}(2 i-1) y_{i}$, and $\iint_{F} y d x d y=\sum_{i=1}^{5} \iint_{F_{i}} y d x d y=\sum_{i=1}^{5} \int_{i=1}^{i}\left[\int_{0}^{y_{i}} y d y\right] d x=\frac{1}{2} \sum_{i=1}^{5} y_{i}^{2}$.

Therefore, Formulas (1) are transformed into the following form:

$$
\begin{aligned}
& x_{c}=\frac{1}{2}\left(\frac{y_{1}+3 y_{2}+5 y_{3}+7 y_{4}+9 y_{5}}{y_{1}+y_{2}+y_{3}+y_{4}+y_{5}}\right), \\
& y_{c}=\frac{1}{2}\left(\frac{y_{1}^{2}+y_{2}^{2}+y_{3}^{2}+y_{4}^{2}+y_{5}^{2}}{y_{1}+y_{2}+y_{3}+y_{4}+y_{5}}\right) .
\end{aligned}
$$

Normalizing our fuzzy data by dividing each $\mathrm{m}(\mathrm{x}), \mathrm{x} \in \mathrm{U}$, with the sum of all membership degrees we can assume without loss of the generality that $y_{1}+y_{2}+y_{3}+y_{4}+y_{5}=1$. Therefore, we can write:

$$
\begin{aligned}
& x_{c}=\frac{1}{2}\left(y_{1}+3 y_{2}+5 y_{3}+7 y_{4}+9 y_{5}\right), \\
& y_{c}=\frac{1}{2}\left(y_{1}^{2}+y_{2}^{2}+y_{3}^{2}+y_{4}^{2}+y_{5}^{2}\right)
\end{aligned}
$$

with $y_{i}=\frac{m\left(x_{i}\right)}{\sum_{x \in U} m(x)}$, where $\mathrm{x}_{1}=\mathrm{a}, \mathrm{x}_{2}=\mathrm{b}, \mathrm{x}_{3}=\mathrm{c}, \mathrm{x}_{4}=\mathrm{d}$ and $\mathrm{x}_{5}=\mathrm{e}$.

But $0 \leq\left(\mathrm{y}_{1}-\mathrm{y}_{2}\right)^{2}=\mathrm{y}_{1}{ }^{2}+\mathrm{y}_{2}{ }^{2}-2 \mathrm{y}_{1} \mathrm{y}_{2}$, therefore $\mathrm{y}_{1}{ }^{2}+\mathrm{y}_{2}{ }^{2} \geq 2 \mathrm{y}_{1} \mathrm{y}_{2}$, with the equality holding if, and only if, $\mathrm{y}_{1}=\mathrm{y}_{2}$. In the same way one finds that $\mathrm{y}_{1}{ }^{2}+\mathrm{y}_{3}{ }^{2} \geq 2 \mathrm{y}_{1} \mathrm{y}_{3}$, and so on. Hence it is easy to check that $\left(\mathrm{y}_{1}+\mathrm{y}_{2}+\mathrm{y}_{3}+\mathrm{y}_{4}+\mathrm{y}_{5}\right)^{2} \leq 5\left(\mathrm{y}_{1}^{2}+\mathrm{y}_{2}^{2}+\mathrm{y}_{3}^{2}+\mathrm{y}_{4}^{2}+\mathrm{y}_{5}^{2}\right)$, with the equality holding if, and only if $\mathrm{y}_{1}$ $=\mathrm{y}_{2}=\mathrm{y}_{3}=\mathrm{y}_{4}=\mathrm{y}_{5}$. However, $\mathrm{y}_{1}+\mathrm{y}_{2}+\mathrm{y}_{3}+\mathrm{y}_{4}+\mathrm{y}_{5}=1$; therefore, $1 \leq 5\left(\mathrm{y}_{1}{ }^{2}+\mathrm{y}_{2}{ }^{2}+\mathrm{y}_{3}{ }^{2}+\mathrm{y}_{4}{ }^{2}+\mathrm{y}_{5}{ }^{2}\right)(3)$, with the equality holding if, and only if $\mathrm{y}_{1}=\mathrm{y}_{2}=\mathrm{y}_{3}=\mathrm{y}_{4}=\mathrm{y}_{5}=\frac{1}{5}$. In this case the first of Formulas (2) gives that $x_{c}=\frac{5}{2}$.

Further, combining the Inequality (3) with the second of Formulas (2) one finds that $1 \leq 10 y_{c}$, or $y_{c} \geq \frac{1}{10}$. Therefore, the unique minimum for $y_{c}$ corresponds to the center of gravity $F_{m}\left(\frac{5}{2}, \frac{1}{10}\right)$. 
The ideal case is when $y_{1}=y_{2}=y_{3}=y_{4}=0$ and $y_{5}=1$. Then from Formulas (2) we get that $x_{c}=\frac{9}{2}$ and $y_{c}=\frac{1}{2}$. Therefore the center of gravity in the ideal case is the point $F_{i}\left(\frac{9}{2}, \frac{1}{2}\right)$. On the other hand the worst case is when $y_{1}=1$ and $y_{2}=y_{3}=y_{4}=y_{5}=0$. Then for formulas (2) we find that the center of gravity is the point $F_{w}\left(\frac{1}{2}, \frac{1}{2}\right)$. Thus, the "area" where the center of gravity $F_{c}$ lies is represented by the triangle $\mathrm{F}_{\mathrm{w}} \mathrm{F}_{\mathrm{m}} \mathrm{F}_{\mathrm{i}}$ of Figure 2 .

Figure 2. Graphical representation of the "area" of the center of gravity.

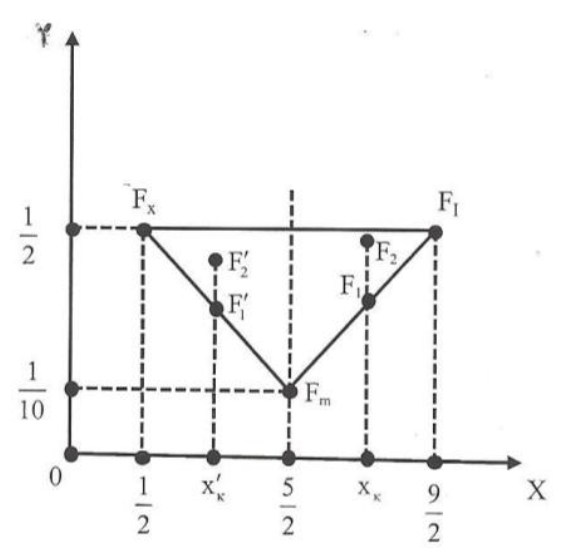

Then from elementary geometric considerations it follows that for two groups of students with the same $x_{c} \geq 2.5$ the group having the center of gravity which is situated closer to $F_{i}$ is the group with the higher $y_{c}$; and for two groups with the same $x_{c}<2.5$ the group having the center of gravity which is situated farther to $F_{w}$ is the group with the lower $y_{c}$. Based on the above considerations we formulate our criterion for comparing the groups' performances as follows:

- Among two or more groups the group with the biggest $x_{c}$ performs better.

- If two or more groups have the same $x_{c} \geq 2.5$, then the group with the higher $y_{c}$ performs better.

- If two or more groups have the same $x_{c}<2.5$, then the group with the lower $y_{c}$ performs better.

\subsection{The Group's Uncertainty}

It is well known that the amount of information obtained by an action can be measured by the reduction of uncertainty resulting from this action. Accordingly a system's uncertainty is connected to its capacity in obtaining relevant information. Therefore, a measure of uncertainty could be adopted as an alternative defuzzification technique for the student groups' assessment model developed in the previous section.

Within the domain of possibility theory uncertainty consists of strife (or discord), which expresses conflicts among the various sets of alternatives, and non-specificity (or imprecision), which indicates that some alternatives are left unspecified, i.e., it expresses conflicts among the sizes (cardinalities) of the various sets of alternatives ([14]; p. 28).

Strife is measured by the function $S T(r)$ on the ordered possibility distribution

$\mathrm{r}: \mathrm{r}_{1}=1 \geq \mathrm{r}_{2} \geq \ldots \ldots . \mathrm{r}_{\mathrm{rn}} \geq \mathrm{r}_{\mathrm{rn}+1}$ of a group of students defined by 


$$
S T(r)=\frac{1}{\log 2}\left[\sum_{i=2}^{m}\left(r_{i}-r_{i+1}\right) \log \frac{i}{\sum_{j=1}^{i} r_{j}}\right]
$$

Similarly, non-specificity is measured by the function $N(r)=\frac{1}{\log 2}\left[\sum_{i=2}^{m}\left(r_{i}-r_{i+1}\right) \log i\right]$

The sum $T(r)=S T(r)+N(r)$ is a measure of the total possibilistic uncertainty for ordered possibility distributions. The lower is the value of $\mathrm{T}(\mathrm{r})$, which means greater reduction of the initially existing uncertainty, the better the system's performance.

We must emphasize that the two defuzzification methods presented above treat differently the idea of a group's performance. In fact, the weighted average plays the main role in the centroid method, i.e., the result of the group's performance close to its ideal performance has much more weight than the one close to the lower end. In other words, while the measure of uncertainty is dealing with the average group's performance, the "centroid" method is mostly looking at the quality of the performance. Consequently, some differences could appear in evaluating a group's performance by these two different approaches. Therefore, it is argued that a combined use of them could help the user in finding the ideal profile of the group's performance according to his/her personal criteria of goals.

There are also other defuzzification techniques in use, such as the calculation of the group's total probabilistic uncertainty, i.e., the classical Shannon's entropy expressed in terms of the Dempster-Shafer mathematical theory of evidence for use in a fuzzy environment (e.g., see [4]), the calculation of the group's ambiguity which is a generalization of the Shannon's entropy in possibility theory that captures both strife and non specificity (e.g., see [15]), etc.

Next we give an example illustrating our results in practice.

EXAMPLE: The following data was obtained by assessing the mathematical skills of two groups of students of the Technological Educational Institute of Patras, Greece being at their first term of studies:

First group (35 students from the School of Technological Applications, i.e., future engineers)

$$
\begin{gathered}
A_{11}=\left\{(a, 0),(b, 0),(c, 0.5),(d, 0.25),(e, 0 . .25), A_{12}=\{(a, 0),(b, 0),(c, 0.5),(d, 0.25),(e, 0)\},\right. \\
A_{13}=\{(a, 0.25),(b, 0.25),(c, 0.25),(d, 0),(e, 0)\}
\end{gathered}
$$

According to the above notation the first index of $A_{i j}$ denotes the group $(i=1,2)$ and the second index denotes the corresponding students' characteristic $S_{j}(j=1,2,3)$. We calculated the membership degrees of the $5^{3}$ (ordered samples with replacement of 3 objects taken from 5) in total possible students' profiles as it is described in Section 2 (see column of $m_{s}(1)$ in Table 1). For example, for the profile $s=(c, c, a)$ one finds that $m_{s}=0.5 \times 0.5 \times 0.25=0.06225$. From the values of the column of $\mathrm{m}_{\mathrm{s}}(1)$ it turns out that the maximal membership degree of students' profiles is 0.06225 . Therefore, the possibility of each $s$ in $U^{3}$ is given by $r_{s}=\frac{m_{s}}{0.06225}$. The possibilities of the students' profiles are presented in column of $r_{s}(1)$ of Table 1 . One could also calculate the probabilities of the students' profiles using the formula for $p_{s}$ given in section 2. However, according to Shackle [16] and many other researchers after him, human cognition is better presented by possibility rather than by probability theory. Therefore, adopting this view, we considered that the calculation of the probabilities was not necessary. 
Table 1. Profiles with non zero membership degrees.

\begin{tabular}{ccccccccc}
\hline $\mathbf{A}_{\mathbf{1}}$ & $\mathbf{A}_{\mathbf{2}}$ & $\mathbf{A}_{\mathbf{3}}$ & $\boldsymbol{m}_{\mathbf{s}}(\mathbf{1})$ & $\boldsymbol{r}_{\mathbf{s}}(\mathbf{1})$ & $\boldsymbol{m}_{\boldsymbol{s}}(\mathbf{2})$ & $\boldsymbol{r}_{\mathrm{s}}(\mathbf{2})$ & $\boldsymbol{f}(\mathbf{s})$ & $\boldsymbol{r}(\mathbf{s})$ \\
\hline $\mathrm{b}$ & $\mathrm{b}$ & $\mathrm{b}$ & 0 & 0 & 0.016 & 0.258 & 0.016 & 0.129 \\
$\mathrm{~b}$ & $\mathrm{~b}$ & $\mathrm{a}$ & 0 & 0 & 0.016 & 0.258 & 0.016 & 0.129 \\
$\mathrm{~b}$ & $\mathrm{a}$ & $\mathrm{a}$ & 0 & 0 & 0.016 & 0.258 & 0.016 & 0.129 \\
$\mathrm{c}$ & $\mathrm{c}$ & $\mathrm{c}$ & 0.062 & 1 & 0.062 & 1 & 0.124 & 1 \\
$\mathrm{c}$ & $\mathrm{c}$ & $\mathrm{a}$ & 0.062 & 1 & 0.062 & 1 & 0.124 & 1 \\
$\mathrm{c}$ & $\mathrm{c}$ & $\mathrm{b}$ & 0 & 0 & 0.031 & 0.5 & 0.031 & 0.25 \\
$\mathrm{c}$ & $\mathrm{a}$ & $\mathrm{a}$ & 0 & 0 & 0.031 & 0.5 & 0.031 & 0.25 \\
$\mathrm{c}$ & $\mathrm{b}$ & $\mathrm{a}$ & 0 & 0 & 0.031 & 0.5 & 0.031 & 0.25 \\
$\mathrm{c}$ & $\mathrm{b}$ & $\mathrm{b}$ & 0 & 0 & 0.031 & 0.5 & 0.031 & 0.25 \\
$\mathrm{~d}$ & $\mathrm{~d}$ & $\mathrm{a}$ & 0.016 & 0.258 & 0 & 0 & 0.016 & 0.129 \\
$\mathrm{~d}$ & $\mathrm{~d}$ & $\mathrm{~b}$ & 0.016 & 0.258 & 0 & 0 & 0.016 & 0.129 \\
$\mathrm{~d}$ & $\mathrm{~d}$ & $\mathrm{c}$ & 0.016 & 0.258 & 0 & 0 & 0.016 & 0.129 \\
$\mathrm{~d}$ & $\mathrm{a}$ & $\mathrm{a}$ & 0 & 0 & 0.016 & 0.258 & 0.016 & 0.129 \\
$\mathrm{~d}$ & $\mathrm{~b}$ & $\mathrm{a}$ & 0 & 0 & 0.016 & 0.258 & 0.016 & 0.129 \\
$\mathrm{~d}$ & $\mathrm{~b}$ & $\mathrm{~b}$ & 0 & 0 & 0.016 & 0.258 & 0.016 & 0.129 \\
$\mathrm{~d}$ & $\mathrm{c}$ & $\mathrm{a}$ & 0.031 & 0.5 & 0.031 & 0.5 & 0.062 & 0.5 \\
$\mathrm{~d}$ & $\mathrm{c}$ & $\mathrm{b}$ & 0.031 & 0.5 & 0.031 & 0.5 & 0.062 & 0.5 \\
$\mathrm{~d}$ & $\mathrm{c}$ & $\mathrm{c}$ & 0.031 & 0.5 & 0.031 & 0.5 & 0.062 & 0.5 \\
$\mathrm{e}$ & $\mathrm{c}$ & $\mathrm{a}$ & 0.031 & 0.5 & 0 & 0 & 0.031 & 0.25 \\
$\mathrm{e}$ & $\mathrm{c}$ & $\mathrm{b}$ & 0.031 & 0.5 & 0 & 0 & 0.031 & 0.25 \\
$\mathrm{e}$ & $\mathrm{c}$ & $\mathrm{c}$ & 0.031 & 0.5 & 0 & 0 & 0.031 & 0.25 \\
$\mathrm{e}$ & $\mathrm{d}$ & $\mathrm{a}$ & 0.016 & 0.258 & 0 & 0 & 0.016 & 0.129 \\
$\mathrm{e}$ & $\mathrm{d}$ & $\mathrm{b}$ & 0.016 & 0.258 & 0 & 0 & 0.016 & 0.129 \\
$\mathrm{e}$ & $\mathrm{d}$ & $\mathrm{c}$ & 0.016 & 0.258 & 0 & 0 & 0.016 & 0.129 \\
\hline & & & 0.016 &
\end{tabular}

The outcomes of Table 1 are with accuracy up to the third decimal point.

Second group (50 students from the School of Management and Economics).

$$
\begin{gathered}
A_{21}=\{(a, 0),(b, 0.25),(c, 0.5),(d, 0.25),(e, 0)\}, A_{22}=\{(a, 0.25),(b, 0.25),(c, 0.5),(d, 0),(e, 0)\} \\
A_{23}=\{(a, 0.25),(b, 0.25),(c, 0.25),(d, 0),(e, 0)\} .
\end{gathered}
$$

The membership degrees and the possibilities of students' profiles are presented in columns of $\mathrm{m}_{\mathrm{s}}(2)$ and $\mathrm{r}_{\mathrm{s}}(2)$ of Table 1 respectively.

In order to study the combined results of the two groups' performance we also calculated the pseudo-frequencies $f(s)=m_{s}(1)+m_{s}(2)$ and the combined possibilities of all profiles presented in the last two columns of Table 1 .

We compare now the two groups' performance by applying the centroid method. For the first characteristic (knowledge of the subject matter) we have:

$$
A_{11}=\left\{(a, 0),(b, 0),(c, 0.5),(d, 0.25),(e, 0.25), A_{21}=\{(a, 0),(b, 0.25),(c, 0.5),(d, 0.25),(e, 0)\}\right.
$$

and respectively

$$
x_{c 11}=\frac{1}{2}(5 \times 0.5+7 \times 0.25+9 \times 0.25)=3.25, x_{c 21}=\frac{1}{2}(3 \times 0.25+5 \times 0.5+7 \times 0.25)=2.25
$$


Thus, by our criterion the first group demonstrates better performance.

For the second characteristic (problem solving abilities) we have:

$$
A_{12}=\{(a, 0),(b, 0),(c, 0.5),(d, 0.25),(e, 0)\}, A_{22}=\{(a, 0.25),(b, 0.25),(c, 0.5),(d, 0),(e, 0)\} .
$$

Normalizing the membership degrees in the first of the above fuzzy subsets of $U(0.5: 0.75 \approx 0.67$ and 0.25:0.75 $\approx 0.33)$ we get $\mathrm{A}_{12}=\{(\mathrm{a}, 0),(\mathrm{b}, 0),(\mathrm{c}, 0.67),(\mathrm{d}, 0.33),(\mathrm{e}, 0)\}$. Therefore

$$
x_{c 12}=\frac{1}{2}(5 \times 0.67+7 \times 0.33)=2.83, x_{c 22}=\frac{1}{2}(0.25+3 \times 0.25+5 \times 0.25)=1.125
$$

By our criterion, the first group again demonstrates a significantly better performance.

Finally, for the third characteristic (analogical reasoning) we have

$$
A_{13}=A_{23}=\{(a, 0.25),(b, 0.25),(c, 0.25),(d, 0),(e, 0)\},
$$

which obviously means that in this case the performances of both groups are identical.

Based on our calculations we can conclude that the first group demonstrated a significantly better performance concerning the knowledge of the subject matter and problem solving, but performed identically with the second one concerning analogical reasoning.

Calculating the possibilities of all profiles (column of $r_{s}(1)$ in Table 1) one finds that the ordered possibility distribution for the first student group is:

$$
\begin{aligned}
r: r_{1}=r_{2}=1, r_{3}=r_{4}=r_{5}= & r_{6}=r_{7}=r_{8}=0,5, r_{9}=r_{10}=r_{11}=r_{12}=r_{13}=r_{14}=0,258, \\
& r_{15}=r_{16}=\ldots \ldots .=r_{125}=0 .
\end{aligned}
$$

Thus, with the help of a calculator one finds that

$$
\begin{aligned}
S T(r)=\frac{1}{\log 2}\left[\sum _ { i = 2 } ^ { 1 4 } \left(r_{i}\right.\right. & \left.\left.-r_{i+1}\right) \log \frac{i}{\sum_{j=1}^{i} r_{j}}\right] \approx \frac{1}{0.301}\left(0.5 \log \frac{2}{2}+0.242 \log \frac{8}{5}+0.258 \log \frac{14}{6.548}\right) \approx \\
& \approx(3.32) .(0.242) .(0.204)+(0.258) .0 .33 \approx 0.445 .
\end{aligned}
$$

Also the group's non-specificity is $N(r)=\frac{1}{\log 2}\left[\sum_{i=2}^{14}\left(r_{i}-r_{i+1}\right) \log i\right]$

$\approx 3.32(0.5 \log 2+0.242 \log 8+0.258 \log 14) \approx 2.208$. Therefore we finally have that $\mathrm{T}(\mathrm{r}) \approx 2,653$

The ordered possibility distribution for the second student group (column of $r_{s}(2)$ in Table 1) is:

$$
\mathrm{r}: \mathrm{r}_{1}=\mathrm{r}_{2}=1, \mathrm{r}_{3}=\mathrm{r}_{4}=\mathrm{r}_{5}=\mathrm{r}_{6}=\mathrm{r}_{7}=\mathrm{r}_{8}=0,5, \mathrm{r}_{9}=\mathrm{r}_{10}=\mathrm{r}_{11}=\mathrm{r}_{12}=\mathrm{r}_{13}=0,258, \mathrm{r}_{14}=\mathrm{r}_{15}=\ldots \ldots=\mathrm{r}_{125}=0
$$

and working in the same way as above one finds that $\mathrm{T}(\mathrm{r})=0.432+2.179=2.611$.

Therefore, since $2.611<2.653$, it turns out that the second group had in general a slightly better average performance than the first one. In contrast, according to the results of the centroid defuzzification method the first group demonstrated a significantly better performance concerning the knowledge of the subject mater and the problem solving and performed identically with the second one concerning the analogical reasoning. The reasons causing these differences have already been explained above. 


\section{Students' Individual Assessment}

The outputs of our fuzzy model developed above can be used not only for assessing the performance of student groups', but also for the students' individual assessment. In fact, if $n=1$ (we recall that $n$ denotes the number of students' of the group under study), then from the definition of the membership function $m$ given in Section 2 it becomes evident that in each $A_{\mathrm{i}}, \mathrm{i}=1,2,3$, there exists a unique element $x$ of $U$ with membership degree 1 , while all the others have membership degree 0 . The centroid method is trivially applicable in this marginal case.

For example, if $A_{11}=\{(a, 0),(b, 0),(c, 0),(d, 1),(e, 0)\}$ and $A_{21}=\{(a, 0),(b, 0),(c, 1),(d, 0),(e, 0)\}$, then obviously the first student demonstrates a better performance with respect to the knowledge acquisition (characteristic $S_{1}$ ). This is crossed by the centroid method, since $x_{c 11}=\frac{7}{2}$ and $x_{c 21}=\frac{5}{2}$.

As a consequence of the above situation $(n=1)$, there exists a unique student profile $s$ with $m_{s}=1$, while all the others have membership degree 0 . In other words, each student is characterized in this case by a unique profile, which gives us the requested information about his/her performance. For example, if $(c, b, a)$ and $(c, b, b)$ are the characteristic profiles for students $x$ and $y$ respectively, then clearly $y$ demonstrates a better performance than $x$. In contrast, if $(d, b, b)$ and $(c, c, b)$ are the corresponding profiles, then $x$ demonstrates a better performance than $y$ concerning the knowledge acquisition, but $y$ demonstrates a better performance than $x$ concerning the problem solving skills (characteristic $S_{2}$ ). Mathematically speaking this means that the students' characteristic profiles define a relationship of partial order among students' with respect to their performance.

A. Jones developed a fuzzy model to the field of Education involving several theoretical constructs related to assessment, amongst which is a technique for assessing the deviation of a student's knowledge with respect to the teacher's knowledge, which is taken as a reference [17,18]. Here we shall present this technique, properly adapted with respect to our fuzzy model, as an alternative fuzzy method for the students' individual assessment.

Let $X=\left\{S_{1}, S_{2}, S_{3}\right\}$ be the set of the students' characteristics under assessment that we have considered in section 2 . Then a fuzzy subset of $X$ of the form $\left\{\left(S_{1}, m\left(S_{1}\right)\right),\left(S_{2}, m\left(S_{2}\right)\right),\left(S_{3}, m\left(S_{3}\right)\right\}\right.$ can be assigned to each student, where the membership function $m$ takes the values $0,0.25,0.5,0.75,1$ according to the level of the student's performance. The teacher's fuzzy measurement is always equal to 1 , which means that the fuzzy subset of $X$ corresponding to the teacher is $\left\{\left(S_{1}, 1\right),\left(S_{2}, 1\right),\left(S_{3}, 1\right)\right\}$.

Then the fuzzy deviation of the student $\mathrm{i}$ with respect to the teacher is defined to be the fuzzy subset $D_{i}=\left\{\left(S_{1}, 1-m\left(S_{1}\right)\right),\left(S_{2}, 1-m\left(S_{2}\right)\right),\left(S_{3}, 1-m\left(S_{3}\right)\right\}\right.$ of $X$.

This assessment by reference to the teacher provides us with the ideal student as the one with nil deviation in all his/her components and it defines a relationship of partial order among students'. The following example illustrates this theoretical framework in practice.

EXAMPLE: We reconsider the group of 35 students of the School of Technological Applications of the Technological Educational Institute of Patras, Greece of our example of section 2. In assessing the students' individual performance by applying the A. Jones technique we found the following types of deviations with respect to the teacher:

$$
\begin{aligned}
& D_{1}=\left\{\left(S_{1}, 0.75\right),\left(S_{2}, 0.75\right),\left(S_{3}, 1\right)\right\} \text { (this type of deviation was related with } 2 \text { students) } \\
& D_{2}=\left\{\left(S_{1}, 0.5\right),\left(S_{2}, 1\right),\left(S_{3}, 1\right)\right\} \text { (related with } 7 \text { students) }
\end{aligned}
$$


$D_{3}=\left\{\left(S_{1}, 0.5\right),\left(S_{2}, 0.75\right),\left(S_{3}, 1\right)\right\}$ (related with 5 students)

$D_{4}=\left\{\left(S_{1}, 0.5\right),\left(S_{2}, 0.75\right),\left(S_{3}, 0.75\right)\right\}$ (related with 4 students)

$D_{5}=\left\{\left(S_{1}, 0.25\right),\left(S_{2}, 0.5\right),\left(S_{3}, 0.75\right)\right\}$ (related with 3 students)

$D_{6}=\left\{\left(S_{1}, 0.25\right),\left(S_{2}, 0.25\right),\left(S_{3}, 0.5\right)\right\}$ (related with 6 students)

$D_{7}=\left\{\left(S_{1}, 0\right),\left(S_{2}, 0.5\right),\left(S_{3}, 0.75\right)\right\}$ (related with 1 student)

$D_{8}=\left\{\left(S_{1}, 0\right),\left(S_{2}, 0.5\right),\left(S_{3}, 0.5\right)\right\}$ (related with 2 students)

$D_{9}=\left\{\left(S_{1}, 0\right),\left(S_{2}, 0.25\right),\left(S_{3}, 0.5\right)\right\}$ (related with 1 student)

$D_{10}=\left\{\left(S_{1}, 0\right),\left(S_{2}, 0.25\right),\left(S_{3}, 025\right)\right\}$ (related with 3 students)

$D_{11}=\left\{\left(S_{1}, 0\right),\left(S_{2}, 0\right),\left(S_{3}, 0.25\right)\right\}$ (related with 1 student)

On comparing the above types of students' deviations it becomes evident that the students possessing the type $D_{3}$ of deviation demonstrate a better performance than those possessing the type $D_{1}$, the students possessing the type $D_{4}$ demonstrate a better performance than those possessing the type $D_{3}$ and so on. However, the students possessing the type $D_{1}$ demonstrate a better performance with respect to problem solving than those possessing the type $D_{2}$, who demonstrate a better performance with respect to the knowledge acquisition. Similarly, the students possessing the type $D_{6}$ demonstrate a better performance with respect to problem solving and analogical reasoning than those possessing the type $D_{7}$, who demonstrate a better performance with respect to the knowledge acquisition. In other words, this type of assessment by reference to the teacher defines a relationship of partial order among students' with respect to their performance.

Notice that the teacher may put a target for his/her class and may establish didactic strategies in order to achieve it. For example he/she may ask for the deviation, say d, to be $0.25 \leq d \leq 0.5$, for all students and all characteristics. The above fuzzy framework could help him/her to determine the divergences with respect to this target and hence readapt his/her didactic plans in order to diminish them.

\section{Conclusions and Discussion}

The following conclusions can be drawn from those presented in this paper:

- Fuzzy logic, due to its nature of including multiple values, offers a wider and richer field of resources for assessing the students' performance than the classical crisp characterization does by assigning a mark to each student, expressed either with a numerical value within a given scale or with a letter corresponding to the percentage of the student's success.

- In this article we developed a fuzzy model for assessing student groups' knowledge and skills, in which the students' characteristics under assessment are represented as fuzzy subsets of a set of linguistic labels characterizing their performance.

- The group's total possibilistic uncertainty and the coordinates of the center of gravity of the graph of the membership function involved were used as defuzzification methods in converting our fuzzy outputs to a crisp number.

- Techniques of assessing the students' performance individually were also discussed and examples were presented illustrating the use of our results in practice. 
Our model is actually a proper adaptation of a more general fuzzy model developed in earlier papers to represent in an effective way a system's operation in situations characterized by a degree of vagueness and/or uncertainty. Our plans for future research include among the others the possible extension of this model for the description of more such situations in Education and in other human activities (human cognition, artificial intelligence, management, etc.).

\section{Conflicts of Interest}

The author declares no conflict of interest.

\section{References}

1. Zadeh, L.A. Fuzzy sets. Inf. Control 1965, 8, 338-353.

2. Zadeh, L.A. Fuzzy algorithms. Inf. Control 1968, 12, 94-102.

3. Voskoglou, M.G. Stochastic and Fuzzy Models in Mathematics Education, Artificial Intelligence and Management; Lambert Academic Publishing: Saarbrucken, Germany, 2011.

4. Voskoglou, M.G. A study on fuzzy systems. Am. J. Comput. Appl. Math. 2012, 2, 232-240.

5. Voskoglou, M.G. Fuzzy logic and uncertainty in mathematics education. Int. J. Appl. Fuzzy Sets Artif. Intell. 2011, 1, 45-64.

6. Voskoglou, M.G. A fuzzy model for human reasoning. Int. J. Math. Eng. Comput. 2012, 3, $61-71$.

7. Klir, G.J.; Folger, T.A. Fuzzy Sets, Uncertainty and Information; Prentice-Hall: London, UK, 1988.

8. Van Broekhoven, E.; de Baets, B. Fast and accurate centre of gravity defuzzification of fuzzy system outputs defined on trapezoidal fuzzy partitions. Fuzzy Sets Syst. 2006, 157, 904-918.

9. Voskoglou, M.G. The process of learning mathematics: A fuzzy set approach. Heuristics Didact. Exact Sci. 1999, 10, 9-13,

10. Subbotin, I.; Badkoobehi, H.; Bilotskii, N. Application of fuzzy logic to learning assessment. Didact. Math. Probl. Investig. 2004, 22, 38-41.

11. Subbotin, I.; Mossovar-Rahmani, F.; Bilotskii, N. Fuzzy logic and the concept of the Zone of Proximate Development. Didact. Math. Probl. Investig. 2011, 36, 101-108.

12. Subbotin, I.; Voskoglou, M.G. Applications of fuzzy logic to case-based reasoning. Int. J. Appl. Fuzzy Sets Artif. Intell. 2011, 1, 7-18.

13. Voskoglou, M.G.; Subbotin, I. Fuzzy models for analogical reasoning. Int. J. Appl. Fuzzy Sets Artif. Intell. 2012, 2, 19-38.

14. Klir, G.J. Principles of Uncertainty: What are they? Why do we mean them? Fuzzy Sets Syst. 1995, 74, 15-31.

15. Perdikaris, S. Using probabilities to compare the intelligence of student groups the van Hiele level theory. J. Math. Sci. Math. Educ. 2012, 7, 27-36.

16. Shackle, G.L.; Decision, S. Order and Time in Human Affairs; Cambridge University Press: Cambridge, United Kingdom, 1961. 
17. Jones, A.; Kaufmman, A.; Zimmerman, H.J. Fuzzy Sets. Theory and Applications; NATO ASI Series, Series C: Mathematical and Physical Sciences; Reidel Publishing Company: Dordrecht, Holland, 1986; Volume 177.

18. Espin, E.A.; Oliveras, C.M.L. Introduction to the use of fuzzy logic in the assessment of mathematics teachers' professional knowledge. Proc. First Mediterr. Conf. Math. 1997, 107-113.

(C) 2013 by the authors; licensee MDPI, Basel, Switzerland. This article is an open access article distributed under the terms and conditions of the Creative Commons Attribution license (http://creativecommons.org/licenses/by/3.0/). 